\title{
The 11th advanced summer school on service-oriented computing
}

\author{
June 25-June 30 2017, Hersonissos, Crete, Greece
}

\section{Guido Wirtz ${ }^{1}$}

(C) Springer-Verlag GmbH Germany 2017

The 11th advanced summer school on service oriented computing (SummerSOC'17) continued a successful series of summer schools that started in 2007, regularly attracting world-class experts in service oriented computing to present state-of-the-art research during a week-long program organized in several thematic tracks: patterns and IoT, formal methods for SOC, computing in the clouds, data science, eHealth and emerging topics. The advanced summer school is regularly attended by top researchers from academia and industry as well as by Ph.D. and graduate students.

During the different sessions at SummerSOC renowned researchers gave invited tutorials on subjects from the themes mentioned above. The afternoon sessions were also dedicated to original research contributions in these areas: accepted contributions were submitted in advance and have been peerreviewed. In addition, the contributions had been extensively discussed during a poster session. The feedback of this discussion too has been folded into the version published in this special issue.

The first article suggests concepts and ideas for a theoretical foundation of service oriented computation, especially the composition of services. The next article is devoted to API governance support through the structural analysis of REST API; the first author received the "SummerSoC Young Researcher Award" sponsored by ICSOC. The following two contributions are from the area of cloud computing and discuss solutions based on the TOSCA standard. Followed by a contribution giving an overview on modeling and execution of data-aware choreographies, the final article introduces an approach on how to partially execute mashup plans during modeling time.
Guido Wirtz

guido.wirtz@uni-bamberg.de

1 Lehrstuhl für Praktische Informatik, Unversität Bamberg, Bamberg, Germany 